\title{
Rassegna
}

\section{Il trattamento delle acque di dialisi: dalla storia ai giorni nostri}

\author{
G. Santorelli, R. Scanziani
}

U.O. Nefrologia e Dialisi, A.O. di Desio e Vimercate, Desio (MB)

Dialysis Water Treatment Systems: From Past to Present

AвSTRACT. In the past dialysis was associated with typical syndromes, such as "hard water syndrome", aluminium-linked encephalopathy and osteomalacia, and pyrogenic reactions. With time it was recognized that water required treatment before being used for haemodialysis. With experience, the type of water for haemodialysis changed from tap to decalcified, then to demineralised, next to osmosis and finally ultra-pure water, the current gold standard.

At present, water treatment systems involve pre-treatment, real treatment and a hydraulic circuit.

The pre-treatment process removes suspended particles which would damage the osmotic membranes. Real treatment prepares water for haemodialysis by using reverse osmosis. The hydraulic circuit distributes osmotic water to the dialysis room and into each machine.

The water treatment systems considered were supplied by Fresenius, Gambro and Farmacastelli. They differ from one another in treatment protocols, construction and disinfection systems.

KEY WoRDS. Water, Dialysis, Osmosis, Treatment

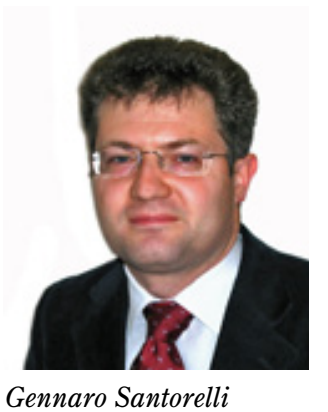

La storia della dialisi si è caratterizzata negli anni per un processo di continua evoluzione e modificazione dei trattamenti e delle relative tecnologie; la Figura 1, ricavata dallo studio di Colton (1), ne illustra le diverse fasi.

Come illustra la Figura, la scelta dell'acqua di dialisi ha subito infatti periodiche variazioni nel tempo, passando dall'acqua di rete all'acqua ultrapura, attraverso le fasi dell'uso dei decalcificatori, dei demineralizzatori, e dell'osmosi semplice.

Quando fu introdotta la dialisi, l'acqua utilizzata per il trattamento era quella potabile proveniente dall'acqua di rete; si ipotizzò infatti che, se questo tipo di acqua poteva essere bevuta dalla popolazione, poteva allo stesso modo essere utilizzata con sicurezza per il trattamento emodialitico (2).

A mano a mano che la dialisi diveniva sempre più diffusa e conosciuta, la letteratura medica si arricchiva di casi di reazioni avverse ad alcune componenti contenute nell'acqua di rete; bisogna infatti considerare il fatto che l'acqua era potabile in quanto ulteriormente trattata con additivi, come il solfato di alluminio o i sali di ferro, sostanze normalmente tossiche per la popolazione in dialisi $(3,4)$.

La presenza di alcuni quadri clinici conclamati nel paziente emodializzato, come la "sindrome dell'acqua dura" (5), l'encefalopatia e l'osteomalacia da alluminio o le reazioni da pirogeni (3), hanno fatto emergere la necessità di trattare e rendere pura l'acqua di rete prima di renderla disponibile come acqua di dialisi.

Tra i contaminanti delle acque di dialisi non vanno dimenticati i batteri e, tra questi, più frequentemente i

\begin{tabular}{|c|c|c|c|c|}
\hline 1944-1959 & $1960-1970$ & \multicolumn{2}{|c|}{ 1971-1989 } & $1990-2000$ \\
\hline Era Antica & Medioevo & \multicolumn{2}{|c|}{ Età Moderna } & Eta Contemporanea \\
\hline Acqua di rete & $\begin{array}{l}\text { Acqus } \\
\text { decakificata }\end{array}$ & Acqua Demineralozzata & Acqua da Osmosi & Acqua Ultrapura \\
\hline \multicolumn{2}{|c|}{ Membrane Cellulosiche } & \multicolumn{3}{|c|}{ Membrane Sintetiche } \\
\hline \multicolumn{2}{|c|}{ Trasporto Diffusivo } & \multicolumn{2}{|c|}{ Trasporto Convettivo } & \\
\hline \multicolumn{2}{|l|}{. } & \multicolumn{3}{|c|}{ Trasporto Misto } \\
\hline
\end{tabular}

Fig. 1 - Evoluzione delle tecnologie e delle metodiche dialitiche. 
batteri gram-negativi e le loro endotossine; già nel 1975 Favero (6) aveva associato la presenza di reazioni intradialitiche da pirogeni al grado di contaminazione del liquido di dialisi.

Inoltre bisogna tener presente che nelle superfici interne delle tubazioni degli impianti di dialisi si può formare naturalmente il biofilm, costituito da microcolonie batteriche inglobate in una matrice extracellulare in grado di rilasciare in circolo pirogeni e batteri (7).

Non esistendo un unico tipo di trattamento purificativo, l'acqua di rete era sottoposta alla combinazione di diversi sistemi di purificazione, a seconda del tipo di contaminazione, del flusso di acqua necessario e del livello di purezza desiderato.

Ad oggi il sistema di trattamento dell'acqua prevede il pre-trattamento, il trattamento vero e proprio e il circuito idraulico per la distribuzione dell'acqua purificata.

Se si considera che un paziente in dialisi viene a contatto con circa 20000 litri di acqua all'anno, ci si rende conto dell'importanza di eliminare dall'acqua le sostanze tossiche e di fornirle caratteristiche di purezza chimica e microbiologica; inoltre è noto che la purezza del liquido di dialisi si associa a un migliore stato nutrizionale del paziente stesso (8).

Verranno ora illustrate separatamente ciascuna delle fasi del sistema di trattamento attualmente utilizzate nei Centri di Dialisi.

\section{Il pre-trattamento}

Il pre-trattamento rimuove le particelle in sospensione e riduce la durezza delle acque, garantendo l'integrità delle membrane dell'osmosi inversa, posta a valle.

Esso comprende:

filtrazione a $20 \mu \mathrm{m}$, con lo scopo di impedire il passaggio di particelle con dimensioni maggiori di $20 \mu \mathrm{m}$; è realizzabile mediante filtro a sabbia o a cartucce filtranti, in base alla quantità di torpidità;

clorazione: ha lo scopo di ridurre il più possibile la carica microbica;

accumulo e rilancio: ha la funzione di consentire la disponibilità di acqua grezza da trattare durante le possibili interruzioni dell'erogazione dell'acqua;

addolcimento: è un procedimento chimico-fisico grazie al quale gli ioni calcio e magnesio vengono sostituiti da ioni sodio. In alternativa qualche ditta propone l'addolcimento mediante iniezione di prodotti chimici "antiscalant" (antiprecipitanti), che hanno lo scopo di tenere in sospensione gli ioni $\mathrm{Ca}^{++} \mathrm{e} \mathrm{Mg}^{++}$. Gli antiprecipitanti sono composti polimerici che aumentano la solubilità dei sali e ne evitano la precipitazione, inoltre il loro peso molecolare è tale per cui vengono totalmente respinti dalle membrane dell'osmosi senza alcuna possibilità di passare nel permeato;

declorazione: è un meccanismo utilizzato per eliminare il cloro precedentemente iniettato, al fine di proteggere l'osmosi inversa. Il sistema più diffuso è realizzato mediante 2 filtri a carbone attivo, preferibilmente installati in serie. Alcune ditte propongono una declorazione mediante iniezione di prodotti chimici (solfiti);

filtrazione a $5 \mathrm{\mu m}$ : ha lo scopo di bloccare le microparticelle con diametro maggiore di $5 \mu \mathrm{m}$, mediante l'utilizzo di filtri a cartuccia $(9,10)$.

\section{Il trattamento vero e proprio}

Il trattamento vero e proprio prevede due alternative: l'osmosi inversa e la deionizzazione.

\section{L'osmosi inversa}

L'osmosi inversa fu introdotta per combattere la tossicità da alluminio, scoperta nella metà degli anni Settanta (7). Tale sistema sfrutta inversamente l'osmosi, cioè il fenomeno naturale che si manifesta quando due soluzioni di diversa concentrazione vengono separate da una membrana semipermeabile, con il passaggio del liquido dalla soluzione più diluita a quella più concentrata. Sfruttando il fenomeno in senso contrario, viene sottoposta la soluzione più concentrata a una pressione superiore a quella osmotica, mediante una pompa ad alta pressione, ottenendo l'inversione del flusso, con il passaggio di acqua pura alla soluzione meno concentrata (Fig. 2), mentre tutti i sali disciolti, le sostanze organiche con PM superiore a 200 , i batteri e i microrganismi vengono respinti $(10,11)$. La membrana osmotica è a spirale avvolta ed è composta da una busta formata da fogli di membrana e da particolari reti di spaziatura, il tutto arrotolato su di un tubo di plastica forato (tubo permeato).

Per effetto della componente radiale della pressione, parte del liquido permea attraverso la membrana, mentre il liquido non permeato viene spinto verso l'uscita della membrana (Fig. 3), raccogliendo ioni, virus, batteri e particelle in sospensione(10).

\section{La deionizzazione}

La deionizzazione è un processo di depurazione dell'acqua di rete basato sull'uso di resine cationiche e anioni- 


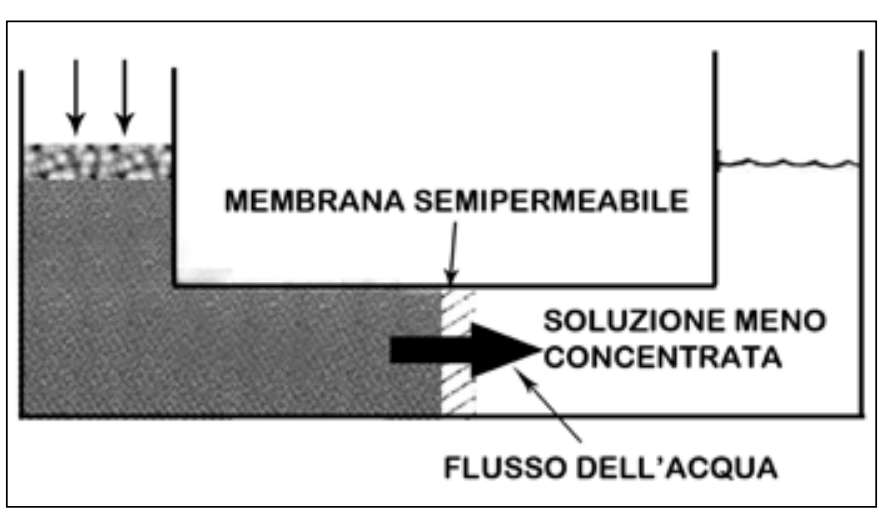

Fig. 2 - Processo dell'osmosi inversa.

che che rimuovono cationi e anioni presenti nell'acqua in scambio con ioni $\mathrm{H}^{+}$e $\mathrm{OH}^{-}$rispettivamente. I deionizzatori sono in grado di produrre l'acqua più pura in termini di contaminazione ionica, ma lasciano invariato il carico biologico (sostanze organiche e microrganismi), oltre a costituire un terreno di crescita per i batteri (3). Nello studio di Laurence (12) viene presa in considerazione l'efficacia di diversi tipi di trattamento dell'acqua partendo da quella di rete; i risultati dello studio evidenziano che il tipo di trattamento che riscuote maggior successo in termini di efficacia è l'osmosi inversa; tale trattamento è utilizzato nel $61 \%$ dei Centri per produrre una qualità di acqua la cui purezza rispondesse alle caratteristiche proposte dal CDC.

Lo studio di Vorbeck-Meister (13) evidenzia che l'osmosi inversa, rispetto alla deionizzazione, è in grado di abbattere in maniera significativa le endotossine nel liquido di dialisi, mentre Thomè (14) dimostra che nei pazienti uremici i valori di proteina $\mathrm{C}$ reattiva diminuiscono quando si passa dalla deionizzazione all'osmosi inversa.

Per tali ragioni, l'osmosi inversa è il processo di trattamento dell'acqua attualmente più utilizzato.

\section{Il circuito idraulico}

Il circuito idraulico per la distribuzione dell'acqua deve mantenere le caratteristiche di purezza dell'acqua; a tal fine deve presentare le seguenti caratteristiche:

- forma ad anello, ad una o più maglie;

- il percorso deve seguire il tragitto più rettilineo e breve possibile;

- la sezione delle tubazioni e la velocità dell'acqua devono consentire un flusso turbolento dell'acqua stessa, perché il flusso laminare favorisce la formazione del biofilm;

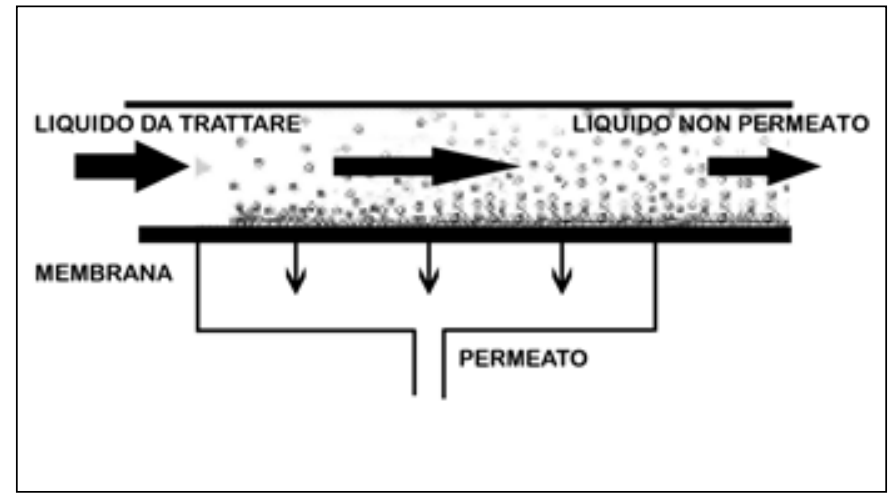

Fig. 3 - Flusso dell'acqua attraverso la membrana.

- la lunghezza della calata (tratto che collega la tubazione del circuito alla valvola di attacco alle macchine) deve essere inferiore a 5- 6 volte il suo diametro, per evitare le zone di ristagno o zone morte (dead-leg).

Nel 1977 il CDC (Centers for Disease Control and Prevention) propone le linee guida per la qualità delle acque per emodialisi, seguite dall'istituzione nel $1981 \mathrm{di}$ quelle dell'AAMI (Association for the Advancement of Medical Instrumentation).

Attualmente le ultime Linee Guida Italiane che abbiamo a disposizione relativamente all'argomento acque e soluzioni per dialisi sono del 2005 e indicano i parametri, limiti, sedi e frequenza di monitoraggio dei contaminanti chimici e microbiologici, sia per l'acqua di rete che per il dialisato (9).

I contaminanti chimici analizzati sono quelli di origine naturale e antropica, nonché i prodotti e sottoprodotti utilizzati per la potabilizzazione; per quanto riguarda $\mathrm{i}$ controlli microbiologici, viene misurata la carica batterica, la presenza di muffe e lieviti e le endotossine. Tali controlli devono essere garantiti con una frequenza semestrale per le analisi chimico-fisiche e ogni 2-4 mesi per quelle microbiologiche (9).

\section{I sistemi di trattamento}

In generale l'intero sistema di trattamento dell'acqua è costituito dall'impianto di dialisi, dal circuito di distribuzione e dai dispositivi di disinfezione.

Limpianto di dialisi comprende un gruppo di accumulo e rilancio dell'acqua greggia, il pre-trattamento, l'osmosi inversa e gli accessori.

L'osmosi inversa ha ottenuto negli anni una notevole 
affidabilità ed efficienza grazie alla realizzazione della cosiddetta bi-osmosi, ottenuta sottoponendo l'acqua da trattare a un doppio passaggio su membrane osmotiche. L'acqua prodotta dalla prima osmosi, definita $1^{\circ}$ stadio, va ad alimentare la seconda, definita $2^{\circ}$ stadio; per ciascuno dei due stadi esistono degli allarmi, in relazione al superamento di valori di conducibilità, che sono espressione di un'alterazione delle membrane delle osmosi stesse, legata alla eccessiva concentrazione di particolari ioni a livello delle osmosi.

Il circuito di distribuzione dell'acqua trattata (loop) può incidere sulla qualità dell'acqua; per tale ragione sono fattori critici la scelta dei materiali, i diametri e il percorso della tubazione, tutti elementi in grado di incidere sullo sviluppo del biofilm.

I dispositivi di disinfezione sono indispensabili per la prevenzione ed eliminazione degli inquinanti biologici.

Verranno ora confrontati tre diversi tipi di sistemi di trattamento, gestiti da tre differenti Aziende: Fresenius, Gambro e Farmacastelli.

\section{FRESENIUS (15)}

L'impianto di dialisi della ditta Fresenius è caratterizzato da: due versioni del pre-trattamento, un sistema di bi-osmosi di tipo evolutivo, il sistema di disinfezione e il circuito di distribuzione.

Il pre-trattamento è disponibile in due versioni, una di tipo standard, con apparecchiature installate a parete, e una di tipo "skid-mounted", con i vari componenti installati in una struttura metallica; entrambe le versioni del pre-trattamento sono applicabili all'osmosi inversa della Fresenius. Il pre-trattamento comprende: la clorazione, la filtrazione, l'addolcimento, la declorazione con utilizzo di carbone attivo e la microfiltrazione ed è conforme alle Linee Guida SIN.

La bi-osmosi (Bi-osmosi AquaB Plus B2) si caratterizza per la presenza di vessel (contenitori delle membrane osmotiche), progettati specificamente per evitare i ristagni e le zone morte e prevenire inquinamenti. Si caratterizza inoltre per la presenza di "break Tank", ovvero un dispositivo che permette di regolare a ogni istante il flusso di acqua scaricato dalla prima osmosi, in base al numero effettivo di monitor in funzione; viene garantita inoltre l'assenza totale di punti di contatto fisico tra l'acqua pretrattata e quella trattata, evitando possibili retrocontaminazioni del circuito.
Il sistema di disinfezione comprende quella chimica a freddo incorporata nella bi-osmosi, quella termica ad acqua calda $\left(90^{\circ} \mathrm{C}\right)$, realizzata con un sistema in linea, "boiler free", e termica a vapore pulito $\left(121^{\circ} \mathrm{C}\right)$.

Il circuito di distribuzione_si caratterizza per l'impiego di materiali alternativi, come l'acciaio inox AISI $316 \mathrm{~L}$ e il PEX (politilene a legami incrociati) (15).

\section{GAMBRO (16)}

Il punto di forza di questo tipo di impianto (CWP) è rappresentato dal sistema di disinfezione termica, detto anche "Chemical free".

L'impianto acqua è composto da: l'osmosi CWP 100 modello WRO ROHH, il pre-trattamento e l'anello di distribuzione della sala dialisi.

Il pre-trattamento comprende: due pre-filtri da $20 \mathrm{mi}-$ cron in parallelo, un sistema di declorazione, un sistema di addolcimento e uno di filtrazione da 5 micron prima dell'ingresso in osmosi; tutti gli elementi del pre-trattamento sono collegati da un tubo in PVC.

L'osmosi inversa è provvista di una unità di acqua calda integrata, definita "unità Riscaldante" in grado di mantenere una temperatura dell'acqua stessa tra $85^{\circ} \mathrm{C}$ e $90^{\circ} \mathrm{C}$, in grado di proteggere l'intero sistema di distribuzione, includendo giornalmente i monitor per dialisi, dalla formazione del biofilm.

La disinfezione avviene ogni notte con ricircolo d'acqua a circa $90{ }^{\circ} \mathrm{C}$; le membrane degli stadi d'osmosi sono disinfettate termicamente 2 volte la settimana.

La linea di distribuzione dell'acqua pura è realizzata in clean PEX (polietilene reticolato) e l'acqua osmotizzata non utilizzata in dialisi viene rinviata all'ingresso della biosmosi in un break-tank .

\section{FARMACASTELLI (17)}

L'impianto è costituito dalle seguenti strutture: filtrazione a sabbia, pretrattamento con dosaggi, biosmosi, sistema di disinfezione, anello di distribuzione. L'elemento caratteristico è il pre-trattamento con consequenziale dosaggio di due soluzioni:

- antiprecipitante

- bisolfito di di sodio

La filtrazione a sabbia è presente tra l'acqua di rete 
ed il serbatoio di stoccaggio e ha lo scopo di eliminare le macroimpurità.

Il pre-trattamento è costituito da:

- Serbatoio di stoccaggio: serbatoio in polietilene, con una capacità adeguata, in grado di garantire autonomia in caso di insufficienza idrica a monte dellimpianto.

- Gruppo di rilancio: è un sistema di pressurizzazione costituito da due elettropompe che lavorano in interscambio automatico, garantendo una pressione adeguata e costante su tutto l'impianto.

- Filtrazione a $20 \mu$ : comprende due filtri a cartuccia in fibre di polipropilene, in grado di trattenere le piccole quantità di particelle sospese della grandezza maggiore di $20 \mu$.

- Antiprecipitanti (o antiscalant); sono in grado di evitare la precipitazione di sali di calcio, magnesio, ma anche ferro.

- Bisolfito di sodio: elimina il cloro residuo ed espleta un'azione batteriostatica sulle membrane osmotiche.

- Filtrazione a $5 \mu$ : è un meccanismo che prevede l'utilizzo di 2 filtri a cartuccia in grado di filtrare e purificare l'acqua prima del suo arrivo all'osmosi; a monte e a valle di ogni filtro sono presenti due manometri e valvole manuali.

Bi-Osmosi: realizzata grazie all'utilizzo di 2 unità osmotiche collegate tra loro in modo da poter operare sia in serie che in parallelo per i casi di emergenze.

Il sistema di disinfezione: caratteristica peculiare è l'utilizzo dell'ozono come alternativa ai sistemi tradizionali di disinfezione; l'ozono è estremamente ossidante ed è letale per batteri, virus, funghi e microrganismi che sopravvivono anche in ambienti ad elevate temperature e acidità.

L'anello di distribuzione è stato realizzato in acciaio INOX con configurazione ad anello chiuso, in grado di garantire una velocità dell'acqua osmotizzata tra 1 e 3 $\mathrm{m} / \mathrm{s}$, al fine di limitare la formazione del biofilm; l'acqua osmotizzata non utilizzata dalle apparecchiature per dialisi rientra nel serbatoio di stoccaggio e quindi ritrattata dall'osmosi inversa.

\section{Conclusioni}

Le caratteristiche del liquido di dialisi rivestono un ruolo fondamentale per il successo a breve e lungo termine di una terapia dialitica.

La qualità del dialisato, sia dal punto di vista chimicofisico che microbiologico, è infatti di estrema importanza per noi nefrologi, indipendentemente dal tipo di trattamento utilizzato; tra l'altro l'adozione delle nuove tecniche emodialitiche come l'emodiafiltrazione online ha ulteriormente posto l'attenzione sull'importanza della purezza delle soluzioni dialitiche.

I mezzi attualmente a disposizione per il monitoraggio delle acque sono le analisi chimico- fisiche e il dosaggio della carica batterica e delle endotossine.

La creazione di tabelle di riferimento ha permesso negli anni di definire i parametri necessari per la valutazione della qualità delle acque, ma la necessità di assicurare un livello di sicurezza sempre più elevato ha imposto un continuo aggiornamento dei valori di riferimento.

$\mathrm{Si}$ rende quindi sempre più necessaria la creazione di nuove Linee guida per definire più sicuri parametri di bontà microbiologica e chimica delle acque di dialisi, al fine di garantire la salvaguardia dei nostri pazienti.

\section{Riassunto}

Nella storia della dialisi, la presenza di alcuni quadri clinici tipici, come la "sindrome dell'acqua dura", l'encefalopatia e l'osteomalacia da alluminio o le reazioni da pirogeni, ha determinato la necessità di trattare l'acqua di rete prima di renderla disponibile per la dialisi; infatti negli anni la scelta dell'acqua per la dialisi ha subito periodiche variazioni passando dall'utilizzo di quella di rete a quella ultrapura, attraverso le fasi di acqua decalcificata, demineralizzata e da osmosi.

Attualmente il trattamento prevede: il pre-trattamento, il trattamento vero e proprio e il circuito idraulico.

Il pre-trattamento ha il compito di rimuovere le particelle in sospensione garantendo l'integrità delle membrane poste a valle. Il trattamento vero e proprio rende l'acqua disponibile per la dialisi e comprende la biosmosi inversa, ottenuta sottoponendo l'acqua da trattare ad un doppio passaggio su membrane osmotiche. Il circuito idraulico ha il compito di distribuire l'acqua osmotizzata in sala dialisi.

Gli Impianti delle Ditte Fresenius, Gambro e Farmacastelli si differenziano tra di loro per le modalità di trattamento dell'acqua, per la struttura degli impianti e i sistemi di disinfezione.

Parole Chiave. Acqua, Dialisi, Osmosi, Trattamento

Si ringraziano i Dottori Gianni Rovinetti della ditta Fresenius, Guido Ravasini della ditta Gambro Hospal, Alessandro Corsi della ditta B. Braun e Sauro Amadei della Farmacastelli per la gentile collaborazione. 
Indirizzo degli Autori:

Dr. Gennaro Santorelli

Via Mazzini 1

20832 Desio (MB)

gennaro.santorelli@aovimercate.org

\section{Bibliografia}

1. Colton CK, Blood Purif1987; 5 (4): 202-51.

2. Favero MS. Good clean water-How dialysis centers treat the vital fluid. Renalife 1987; 2:14-6.

3. Tong MK, Wang W, Kwan T, Chan L, Au T. Water treatment for hemodialysis. Hong Kong J Nephrol 2001; 3(1): 7-14.

4. Pontoriero G, Pozzoni P, Andrulli S. and Locatelli F. The quality of dialysis water. Nephrol Dial Transplant 2003; 18 (7): vii 21-5.

5. Freeman RM, Lawton RL, Chamberlain MA. Hard-water syndrome. N Engl J Med 1967 18; 276 (20): 1113-8.

6. Favero MS, Peterson NJ, Carson LA, Bond WW, Hindman $\mathrm{SH}$. Gram-negative water bacteria in hemodialysis systems. Health Lab Sci 1975; 12 (4): 321-4.

7. Man NK, Degremont A, Darbord JC, et al. Evidence of bacterial biofilm in tubing from hydraulic pathway of he- modialysis system. Artif Organs 1998; 22: 596-600.

8. Schiffl H, Lang S, Stratakis D, Rainad F. Effects of dialysis fluid on nutritional status and inflammatory parameters. Nephrol Dial Transplant 2001, 16: 1683-9.

9. Alloatti S, Bolasco P, Canavese C, et al. Guidelines on water and solutions for dialysis. Italian Society of Nephrology. G Ital Nefrol 2005; 22 (3): 246-73.

10. La dialisi e il Tecnico Sanitario, Montecatini Terme (Pistoia, Italia), Ante, 2004, 109-14.

11. Ledebo I. Purification of Dialysis Fluid: Historical Background and Perspective. Blood Purif 2009; 27 (Suppl 1): 17-9.

12. Laurence RA, Lapierre ST. Quality of Hemodialysis Water: A 7-Year Multicenter study. Am J Kidney Dis 1995; 25 (5): 738-50.

13. Vorbeck-Meister I, Sommer R, Vorbeck F, Horl WH. Quality of water used for hemodialysis: bacteriological and chemical parameters. Nephrol Dial Transplant 1999: 14: 666-75.

14. Thomè FS, Senger M, Garcez C, Garcez J, Chemello C, Manfro RC. Dialysis water treated by reverse osmosis decreases the levels of $\mathrm{C}$-reactive protein in uremic patients. Brazilian Journal of Medical and Biological Research 2005; 38: 789-94.

15. Documentazione Fresenius.

16. Documentazione Gambro.

17. Documentazione Farmacastelli. 